\title{
Criterios GLIM: análisis de su consistencia interna y una comparación con respecto a la valoración global subjetiva
}

\author{
GLIM criteria: Internal consistency analysis and comparison with \\ the subjective global assessment \\ Critérios GLIM: Análise de sua consistência interna e comparação com \\ relação à avaliação subjetiva global
}

\author{
Pilar Navarro ${ }^{1 *}$, Ornella Capelli', Julieta Adaglio', Romina Barritta².
}

Recibido: 1 de junio de 2021. Aceptado para publicación: 10 de septiembre de 2021.

Primero en línea: 23 de septiembre de 2021

https://doi.org/10.35454/rncm.v4n4.304

\section{Resumen \\ Introducción: los criterios Global Leadership Initiative on Malnutrition (GLIM), creados para alcanzar un consenso mundial en cuanto a los criterios diagnós- ticos de la desnutrición (DN) asociada con la enfermedad, requieren ser validados para su uso en la práctica clínica. \\ Objetivo: estimar la consistencia in- terna de los criterios GLIM y compararlos con respecto a la valoración global sub- jetiva (VGS) con el fin de diagnosticar DN en pacientes hospitalizados con riesgo nutricional.}

Métodos: se evaluaron 123 adultos hospitalizados con riesgo de DN (SNAQ $\geq$ 2). Se utilizaron la VGS y GLIM para diagnosticar DN. Se calculó la consistencia interna del GLIM y su concordancia, validez, seguridad y razón de probabilidad con respecto a VGS.

Resultados: la prevalencia de DN fue de 91 \% según GLIM y 88,62 \% según VGS, y fue grave en $52,03 \%$ y $32,52 \%$, respectivamente. La consistencia interna del GLIM resultó aceptable (alfa de Cronbach: 0,6425). La concordancia entre VGS y GLIM fue moderada $(\kappa: 0,5946)$ o buena $(\kappa: 0,7777)$ según la categorización utilizada. GLIM obtuvo una sensibilidad del 99,1\% (intervalo de confianza [IC] $95 \%$ : $95 \%$-100\%) y una especificidad del $71,4 \%$ (IC $95 \%$ : $41,9 \%-91,6 \%)$. El valor predictivo positi-

\section{Summary}

Background: The GLIM (Global Leadership Initiative on Malnutrition) criteria, created to reach a global consensus on diagnostic criteria for disease-related malnutrition (MN), needs to be validated in order to be used in clinical practice.

Objective: To estimate the internal consistency of GLIM criteria and compare it with the Subjective Global Assessment of Nutritional Status (SGA) as a tool for diagnosing malnutrition in hospitalized patients with nutritional risk.

Methods: 123 hospitalized adults and at risk of MN (SNAQ $\geq 2$ ) were assessed. Both SGA and GLIM criteria were used to diagnose MN. The level of concordance, validity, safety and probability ratio between SGA and GLIM were determined.

Results: The prevalence of MN detected by GLIM was $91 \%$ with $52.03 \%$ categorized as severe malnutrition. When using SGA, the prevalence of malnutrition was $88.62 \%$, with $32.52 \%$ detected as severe. The internal consistency of GLIM was acceptable (Cronbach's alpha: 0.6425). Agreement between tools was moderate ( $\kappa: 0.5946)$ or good $(\kappa: 0,7777)$ according to the categorization used. When compared to SGA, sensitivity and specificity of the GLIM criteria were $99.1 \%$ (95\% CI 95-100\%) and $71.4 \%$ (95\% Cl $41.9-91.6 \%)$, respectively. The positive and negative likelihood

\section{Resumo}

Introdução: Os critérios Global Leadership Initiative on Malnutrition (GLIM), criados para alcançar um consenso mundial respeito dos critérios diagnósticos da desnutrição associada à doença (DN), requerem validação para seu uso na prática clínica.

Objetivo: Estimar a consistência interna dos critérios GLIM e compará-los com a Avaliação Subjetiva Global (ASG) com o objetivo de diagnosticar DN em pacientes hospitalizados com risco nutricional.

Métodos: Foram avaliados 123 adultos hospitalizados com risco de DN (SNAQ $\geq 2$ ). ASG e GLIM foram usados para diagnosticar DN. Foram calculadas a consistência interna do GLIM e sua concordância, validade, segurança e razão de probabilidade em relação à ASG.

Resultados: A prevalência de DN foi de $91 \%$ pelo GLIM e $88,62 \%$ pela ASG, sendo severa em $52,03 \%$ e $32,52 \%$ respectivamente. A consistência interna do GLIM foi aceitável (Alfa de Cronbach: 0.6425). A concordância entre ASG e GLIM foi moderada $(\kappa: 0,5946)$ ou boa $(\kappa: 0,7777)$ de acordo com a categorização utilizada. 0 GLIM obteve uma sensibilidade de 99,1\% (IC 95\%: 95\%-100\%) e uma especificidade de 71,4\% (IC 95\%: 41,9\%-91,6\%). O valor preditivo positivo foi de $96,4 \%$ (IC $95 \%$ : 91,1-99\%) e o negativo de $90,9 \%$ (IC95\% 
vo fue $96,4 \%$ (IC $95 \%$ : 91,1\%-99\%) y el negativo fue $90,9 \%$ (IC $95 \%$ : 58,7-99,8). La razón de verosimilitud positiva fue 3,47 (IC $95 \%: 1,51-7,94)$ y la negativa fue 0,0128 (IC $95 \%$ : 0,018- 0,929).

Conclusiones: la herramienta GLIM posee una validez justa y concuerda moderadamente con la VGS. Se requieren estudios de validez para su aplicación en la población hospitalaria general.

Palabras clave: GLIM, desnutrición, valoración global subjetiva, diagnóstico nutricional, evaluación nutricional. ratios were $3.47(95 \% \mathrm{Cl} 1.51-7.94)$ and 0.0128 (95\% Cl 0.018-0.929), respectively.

Conclusion: The GLIM diagnostic tool has fair validity and its agreement with SGA was found to be significant. GLIM is a useful tool for diagnosis of malnutrition in hospitalized adults. Further validation studies are required for its application in the general inpatient population.

Keywords: GLIM, malnutrition, Subjective Global Assessment, nutritional diagnostic tool, nutrition assessment.
58,7-99,8). A razão de verossimilhança positiva foi 3,47 (IC 95\%: 1,51-7,94) e negativa 0,0128 (IC 95\%: 0,018-0,929).

Conclusões: A ferramenta GLIM tem uma validade razoável e concorda moderadamente com a ASG. Estudos de validade são necessários para sua aplicação na população hospitalar geral.

Palavras-chave: GLIM, desnutrição, Avaliação subjetiva global, diagnóstico nutricional, avaliação nutricional.
Instituto Universitario Centro de Educación Médica e Investigaciones Clínicas "Norberto Quirno" (IUC). Buenos Aires, Argentina.

2 Centro de Educación Médica e Investigaciones Clínicas "Norberto Quirno" (CEMIC). Buenos Aires, Argentina.
* Correspondencia: Pilar Navarro. pnavarro@cemic.edu.ar

\section{INTRODUCCIÓN}

La desnutrición (DN) hospitalaria es un problema frecuente a nivel mundial cuya prevalencia ha sido ampliamente documentada (19\%-80 \%). En Latinoamérica, la gran prevalencia se asocia con una variedad de complicaciones clínicas, lo cual impone una carga sanitaria y económica. A nivel nacional, la Asociación Argentina de Nutrición Enteral y Parenteral realizó un estudio multicéntrico en 2014 y describió una prevalencia del $48 \%{ }^{(1,2)}$.

El tamizaje nutricional es un método de fácil recolección que debería realizarse en todo paciente en las primeras 24-48 horas de hospitalización para detectar precozmente el riesgo de DN. Esto permitirá implementar tempranamente una adecuada terapia nutricional y, por consiguiente, disminuir el riesgo de complicaciones, estancia hospitalaria y mortalidad. No existe un acuerdo sobre la herramienta de tamizaje a utilizar. Dentro de las herramientas validadas se encuentra el Short Nutritional Assessment Questionnaire (SNAQ), el cual es empleado en nuestro centro por su practicidad ${ }^{(3-5)}$.

En pacientes detectados con riesgo de $\mathrm{DN}$, se debe realizar una evaluación nutricional exhaustiva con el objetivo de diagnosticar DN y determinar su $\operatorname{gravedad}^{(3)}$. A pesar de que varios estudios utilizan la valoración global subjetiva (VGS) como herramienta diagnóstica $^{(6)}$, no existe una herramienta estándar de oro. Debido a la necesidad de un consenso mundial en la definición de los criterios diagnósticos de la DN en el entorno hospitalario, a través de un comité de líderes internacionales se crearon los criterios GLIM (Global Leadership Initiative on Malnutrition). Luego de su lanzamiento, el mismo comité expresó la necesidad de validarlo en la práctica clínica ${ }^{(7)}$. La VGS es considerada en el consenso GLIM como una herramienta "semiestándar de oro" en el diagnóstico de la desnutrición.

El primer estudio a nivel mundial cuyo objetivo fue validar los criterios GLIM utilizando la VGS en pacientes hospitalizados fue publicado por Allard y colaboradores. El estudio concluyó que el GLIM, con respecto a la VGS, subestimó la $\mathrm{DN}$, pero aumentó la probabilidad de que un individuo fuera categorizado como gravemente desnutrido ${ }^{(8)}$. Luego, en 2021, Burgel y colaboradores, y Brito y colaboradores describieron una sensibilidad y especificidad del GLIM mayor del $80 \%$ con respecto a la $\operatorname{VGS}^{(9,10)}$. Ambos consideraron que el GLIM puede ser utilizado en pacientes hospitalizados. Además, Brito y colaboradores concluyeron que la presencia de DN según los criterios GLIM aumenta el riesgo de hospitalizaciones prolongadas y de muerte dentro de los 6 meses siguientes ${ }^{(10)}$.

Hasta el momento, en Latinoamérica son pocos los estudios que abordan la temática que se plantea en la población hospitalaria general. Galindo Martin y colaboradores, a través de un estudio prospectivo descriptivo, demostraron que el diagnóstico de la malnutrición, según el GLIM, es un factor de riesgo para complicaciones a corto plazo como mortalidad intrahospitalaria e ingreso a la unidad de cuidados 
críticos. La disminución de la masa muscular y la inflamación demostraron ser factores de riesgo independientes para las complicaciones ${ }^{(11)}$.

Se presenta la interrogante de si el GLIM es de utilidad para la identificación oportuna y sencilla de la $\mathrm{DN}$ con el fin de implementar el tratamiento nutricional adecuado y evitar las complicaciones asociadas con la misma.

Por lo anteriormente expuesto, en el presente estudio se plantea comparar la herramienta GLIM con la VGS en pacientes hospitalizados en riesgo de DN.

\section{MÉTODOS}

Estudio prospectivo, descriptivo, transversal y de comparación entre dos herramientas de diagnóstico del estado nutricional. El mismo fue realizado en el Hospital Universitario CEMIC (Centro de Educación Médica e Investigaciones Clínicas "Norberto Quirno"), un centro de tercer nivel con 185 camas localizado en Buenos Aires. Se incluyeron pacientes mayores de 18 años, identificados en riesgo de $\mathrm{DN}$ con el uso del método de tamizaje nutricional SNAQ (puntaje $\geq 2$ ), en las unidades de internación de ambas sedes del Hospital Universitario CEMIC entre febrero y marzo de 2020. Se excluyeron pacientes internados en la unidad de cuidados intensivos (UCI), embarazadas y puérperas. Se eliminaron a aquellos pacientes que egresaron de la institución o fallecieron antes de recolectar la totalidad de los datos y pacientes en cuidados paliativos.

El tamaño muestral calculado fue de 120 pacientes, asumiendo un $20 \%$ de pérdida. Se utilizó un promedio de 3 personas por cada ítem de la herramienta para analizar su consistencia interna ${ }^{(12)}$. Según el programa Epidat, con una muestra de 50 pacientes se alcanzaría una potencia del $90 \%$ y se obtendría un coeficiente de correlación de al menos 0,6 . El muestreo fue de tipo no probabilístico por conveniencia ${ }^{(13)}$. Los datos fueron recolectados de forma consecutiva en todos los pacientes con riesgo nutricional al ingreso durante los meses de recolección de datos.

El presente estudio se adecuó a las normas internacionales de investigación de Helsinki de la Asociación Médica Mundial, a la Ley 3301 del Ministerio de Salud del Gobierno de la Ciudad de Buenos Aires, a la Resolución 1480/2011 del Ministerio de Salud de la Nación y a todas las legislaciones y reglamentaciones a las cuales se adhiere el Comité de Ética e Investigación del CEMIC. Luego de ser aprobado por este comité de ética, fue requisito la firma del consentimiento informado previo a la incorporación del individuo al estudio.

\section{Recolección de datos}

Los datos fueron recolectados por licenciados en nutrición previamente capacitados dentro de las primeras 24-48 horas de la admisión hospitalaria del paciente. El mismo encuestador recolectó datos para ambas herramientas. Para disminuir el riesgo intraobservador primero se procedió a la realizar la VGS, ya que la misma incluye componentes subjetivos, y luego se categorizó la DN según los criterios GLIM. Los mismos se obtuvieron, en su mayoría, de fuentes primarias como ser el mismo paciente o sus acompañantes. La fuente secundaria principalmente utilizada fue la historia clínica.

En primer lugar, de haber sido factible, se pesó y midió la altura del individuo con una balanza digital marca $\mathrm{OMROM}^{\odot}$ modelo HN-289 o balanza mecánica de pie $\mathrm{CAM}^{\odot}$ con altímetro de $80-220 \mathrm{~cm}$ de alcance y precisión de $1 \mathrm{~mm}$. Luego se procedió a realizar las herramientas diagnósticas.

\section{Valoración global subjetiva}

Se completaron los 6 dominios que conforman la VGS según la técnica descrita por Detsky y colaboradores a través del interrogatorio y el examen físico. Se categorizó al paciente según el estado nutricional detectado por la herramienta ${ }^{(14,15)}$.

\section{Criterios GLIM}

Se comenzaron a completar los criterios fenotípicos y etiológicos de la herramienta GLIM descritos en la Tabla 1. Se categorizó al paciente con DN con la presencia de al menos 1 criterio fenotípico y 1 criterio etiológico. El criterio fenotípico definió la gravedad de la DN. En cuanto al ítem "masa muscular reducida", se realizó un examen físico y, luego, se utilizaron herramientas complementarias según la edad del paciente:

- Fuerza de agarre: se llevó a cabo en todos los pacientes capaces de realizar la prueba más allá de la edad. Se utilizó el dinamómetro de mano $\left(\mathrm{JAMAR}^{\odot}\right.$ hidráulico modelo 5030J1 con precisión de 90 $\mathrm{kg})$. Se realizaron tres mediciones de cada brazo utilizando el instructivo del dinamómetro y se calculó el promedio de cada uno ${ }^{(16)}$.

- Circunferencia media muscular del brazo (CMMB): Se midió en menores de 75 años, según la descripción de Canicoba M y colaboradores. Se utilizó una cinta métrica inextensible Lufkin $^{\oplus}$ con $1 \mathrm{~mm}$ de precisión y un plicómetro Slim Guide ${ }^{\odot}$ con un rango de medición de 0-85 $\mathrm{mm}$ y precisión de $1 \mathrm{~mm}$. Se 
Tabla 1. Herramienta de diagnóstico nutricional, criterios $\mathrm{GLIM}^{(7)}$

\begin{tabular}{|c|c|c|c|c|c|}
\hline & \multicolumn{3}{|c|}{ Criterios fenotípicos } & \multicolumn{2}{|c|}{ Criterios etiológicos } \\
\hline & Pérdida de peso (\%) & Bajo IMC (kg/m²) & $\begin{array}{l}\text { Masa muscular } \\
\text { reducida* }\end{array}$ & $\begin{array}{l}\text { Ingesta alimentaria (o } \\
\text { absorción) reducida }\end{array}$ & Inflamación \\
\hline $\begin{array}{l}\text { DN } \\
\text { moderada }\end{array}$ & $\begin{array}{l}5 \%-10 \% \text { en los últimos } \\
6 \text { meses o } 10 \% \text { a } 20 \% \text { en } \\
\text { más de } 6 \text { meses }\end{array}$ & $\begin{array}{l}<20 \text { en menores de } \\
70 \text { años o }<22 \text { en } \\
\text { mayores de } 70 \text { años }\end{array}$ & $\begin{array}{l}\text { Déficit leve a } \\
\text { moderado }\end{array}$ & \multirow{2}{*}{$\begin{array}{l}\leq 50 \% \text { del requerimiento } \\
\text { energético, o cualquier } \\
\text { reducción por más de } \\
2 \text { semanas, o cualquier } \\
\text { condición gastrointestinal } \\
\text { que afecte la asimilación/ } \\
\text { absorción de alimentos }\end{array}$} & \multirow{2}{*}{$\begin{array}{l}\text { Enfermedad/ } \\
\text { lesión aguda o } \\
\text { relacionada con } \\
\text { enfermedad } \\
\text { crónica }\end{array}$} \\
\hline DN grave & $\begin{array}{l}>10 \% \text { en los últimos } 6 \\
\text { meses o > } 20 \% \text { en más de } \\
6 \text { meses }\end{array}$ & $\begin{array}{l}<18,5 \text { en menores } \\
\text { de } 70 \text { años o }<20 \text { en } \\
\text { mayores de } 70 \text { años }\end{array}$ & Déficit grave & & \\
\hline \multicolumn{6}{|c|}{$\begin{array}{l}\text { *Se midió la fuerza de agarre, la CMMB, la CP y el examen físico. Se consideraron los siguientes puntos de corte para determinar la } \\
\text { "masa muscular reducida": } \\
\text { - Fuerza de agarre disminuido: }<-2 \text { DE según el sexo y la edad del dinamómetro utilizado }{ }^{(20-23)} \text {. } \\
\text { - CMMB disminuida: leve a moderada entre los percentiles (pc) } 5 \text { y } 10, \text { y grave con } p c<5^{(18)} \text {. } \\
\text { - CP disminuido: } \leq 30,5 \mathrm{~cm}^{(18,19)} \text {. } \\
\text { Para diferenciar entre masa muscular leve/moderadamente reducida y gravemente reducida, en los casos en que las medidas } \\
\text { antropométricas realizadas presenten valores pertenecientes a distintas categorías, se tuvo en cuenta el examen físico del paciente } \\
\text { y la categorización final se realizó según el criterio profesional del propio entrevistador. }\end{array}$} \\
\hline
\end{tabular}

CMMB: circunferencia media muscular del brazo; CP: circunferencia de pantorrilla; DE: desviación estándar; DN: desnutrición; IMC: índice de masa corporal.

emplearon las tablas de referencia NHANES III para calcular los percentiles de los valores según el sexo y la edad ${ }^{(17)}$.

- Circunferencia de pantorrilla (CP): se midió en mayores de 65 años según la técnica descrita por Canicoba y colaboradores, utilizando una cinta métrica inextensible Lufkin ${ }^{\odot}$ con $1 \mathrm{~mm}$ de precisión ${ }^{(18,19)}$.

\section{Análisis de datos}

Los datos recolectados fueron registrados en una base de datos de elaboración propia y analizados empleando el paquete estadístico Stata 11.0 y software VCC Stat Beta 3.0. Para el análisis descriptivo de las variables de caracterización se calculó la frecuencia relativa y para las continuas, la media y la desviación estándar (DE).

La validez interna de los criterios GLIM se estudió por medio de la consistencia interna de los ítems utilizando el alfa de Cronbach. La mayoría de los autores sugiere un margen aceptable para los coeficientes de fiabilidad entre 0,65 y 0,8 , y se consideran valores menores de 0,5 como inaceptables ${ }^{(24-27)}$.

La concordancia se evaluó a través de la prueba kappa $(\kappa)$, la cual comparó las categorías diagnósticas obtenidas a través de VGS y GLIM ("sin DN", "DN moderada" y "DN grave”). Además, se calculó el mismo considerando dos categorías ("sin DN" y "con DN"). La misma se considera muy débil cuando los valores $\kappa$ son inferiores a 0,20 ; débil entre 0,21 y 0,40 ; moderada entre 0,41 y 0,60 ; buena entre 0,61 y 0,80 y muy buena con valores superiores a 0,80 . Se considera el nivel de significancia con valores de $p<0,05^{(28,29)}$.

Se categorizó como "DN" a todos aquellos que pertenecían a las categorías de VGS "B" y "C" y GLIM "DN moderada" y "DN grave". Se calculó la validez de los criterios GLIM para predecir DN mediante la especificidad y sensibilidad según la VGS. La misma se clasificó según los siguientes puntos de corte: "bueno" para ambos valores $>80 \%$, "justo" para uno de los valores $>80 \%$ y ambos $>50 \%$, y "pobre" en caso de al menos un valor $<50 \%{ }^{(30)}$. La seguridad del GLIM fue analizada mediante el valor predictivo positivo (VPP) y valor predictivo negativo (VPN). Por último, se calculó la razón de verosimilitud positiva $(\mathrm{LR}+)$ y negativa (LR -). Se consideraron como de "utilidad altamente relevante" los valores de LR + de 10 y como de "utilidad moderada" los valores entre 5 y 10 . En el caso de LR -, las cifras menores de 0,1 se consideraron como de "utilidad altamente relevante" y entre 0,1-0,2 como de "utilidad moderada"(31-33).

\section{RESULTADOS}

La muestra quedó conformada por 123 pacientes hospitalizados con riesgo de DN. La media de edad fue de 70 años $(\mathrm{DE} \pm 16,26)$ con un mínimo de 20 y un máximo 
de 96 años. Con respecto al sexo, el 55,28 \% fue categorizado como femenino y el $44,72 \%$ como masculino. El $50,41 \%$ de la muestra resultó ser pacientes oncológicos activos y el 30,89\%, pacientes quirúrgicos. En la Tabla 2 se detalla la caracterización de la muestra de estudio.

Tabla 2. Características de la muestra $(n=123)$

\begin{tabular}{|l|c|c|c|}
\hline \multicolumn{1}{|c|}{ Características } & $\mathbf{n}$ & $\%$ & IC $95 \%$ \\
\hline Edad & & & \\
\hline - Menores de 65 años & 29 & 23,57 & $16,59-32,24$ \\
\hline - Mayor o igual a 65 años & 94 & 76,42 & $67,75-83,40$ \\
\hline Sexo & & & \\
\hline - Femenino & 68 & 55,28 & $46,06-64,16$ \\
\hline - Masculino & 55 & 44,72 & $35,83-53,93$ \\
\hline Paciente oncológico & 62 & 50,41 & $41,29-59,48$ \\
\hline Paciente quirúrgico & 38 & 30,89 & $23,04-39,95$ \\
\hline Diagnóstico al ingreso & & & \\
\hline - Sistema digestivo & 28 & 22,7 & $15,89-31,37$ \\
\hline - Sistema nefrourológico & 25 & 20,3 & $13,82-28,72$ \\
\hline - Sistema respiratorio & 19 & 15,4 & $9,78-23,32$ \\
\hline - Sistema osteomuscular & 18 & 14,6 & $9,13-22,41$ \\
\hline - Otros sistemas (hematopoyético, & 34 & 27,6 & $20,14-36,56$ \\
\hline $\begin{array}{l}\text { reproductivo, reumatológico, } \\
\text { nervioso, linfático y metabólico) }\end{array}$ & 14 & 11,38 & $6,59-18,67$ \\
\hline Estado nutricional según VGS & 69 & 56,10 & $46,87-64,93$ \\
\hline - Bien nutrido & 40 & 32,52 & $24,51-41,63$ \\
\hline - DN moderada & & 8,94 & $4,77-15,79$ \\
\hline - DN grave & & $30,48-48,26$ \\
\hline Estado nutricional según GLIM & $42,88-61,05$ \\
\hline - Sin DN & & & \\
\hline - DN moderada & DN grave & & \\
\hline - & 11,02 & \\
\hline
\end{tabular}

En la Tabla 3 se visualizan los diagnósticos según cada herramienta utilizada. La herramienta GLIM mostró una prevalencia de DN del $91 \%$, el $39 \%$ fue categorizado como DN moderada y el 52,03 \% como grave. Con la herramienta VGS se obtuvo $88,6 \%$ de $\mathrm{DN}$, fue moderada en el $56,1 \%$ y grave en el $32,52 \%$.

La herramienta GLIM obtuvo una consistencia interna aceptable, con un valor alfa de Cronbach de
0,6425 . Con respecto a la "correlación del elemento total", la variable que se correlaciona en mayor medida con el alfa del GLIM resultó ser "masa muscular reducida" $(0,8326)$; mientras que "ingesta reducida" $(0,4416)$ lo hizo en menor medida (Tabla 4). El ítem "inflamación" fue eliminado del análisis ya que se observó su presencia en el $100 \%$ de la muestra. La concordancia entre GLIM y VGS fue moderada con un valor de $\kappa: 0,5946$ (intervalo de confianza [IC] $95 \%$ : 0,46-0,52; $p<0,00001$ ) al considerar tres categorías, y fue buena con un valor de $\kappa$ : 0,7777 (IC $95 \%$ : 0,68-0,86; $p<0,00001$ ) al considerar dos categorías de las herramientas.

Tabla 3. Tabla de contingencia 2 x 2 para evaluar la prueba diagnóstica

\begin{tabular}{|l|c|c|c|}
\hline \multirow{2}{*}{ GLIM } & \multicolumn{2}{|c|}{ Patrón de referencia: VGS } & \multirow{2}{*}{ Totales } \\
\cline { 2 - 3 } & $\begin{array}{c}\text { Con DN (B y C): } \\
\text { positivo }\end{array}$ & $\begin{array}{c}\text { Sin DN (A): } \\
\text { negativo }\end{array}$ & \\
\hline Con DN: positivo & 108 & 4 & 112 \\
\hline Sin DN: negativo & 1 & 10 & 11 \\
\hline Totales & 109 & 14 & 123 \\
\hline
\end{tabular}

En la Tabla 5 se pueden apreciar los resultados correspondientes a la validez de los criterios GLIM categorizados como justos. La estimación del LR + mostró una utilidad regular, ya que es 3,47 veces más probable que el GLIM categorice a un paciente desnutrido como tal a que un paciente $\sin \mathrm{DN}$ se categorice como desnutrido. Contrariamente, el LR - mostró una utilidad altamente relevante ya que es 0,01 veces menos probable que un paciente desnutrido sea categorizado como tal a que un paciente sin DN se categorice como desnutrido.

\section{DISCUSIÓN}

El presente estudio es uno de los primeros en Latinoamérica en comparar la VGS con el GLIM en pacientes hospitalizados con riesgo de DN.

Las herramientas diagnósticas fueron aplicadas, tal como se propone en la práctica clínica, únicamente en pacientes con riesgo nutricional, lo que explicaría la alta prevalencia de DN hallada. El SNAQ ha demostrado ser válido y reproducible en pacientes hospitalizados, con una sensibilidad y especificidad mayores del $75 \%$. Es así que no se encuentra exento de haber dejado individuos fuera del estudio que podrían ser desnutridos, por lo que se puede sobreestimar el valor verdadero del comportamiento del $\mathrm{GLIM}^{(34-36)}$. 
Tabla 4. Resultados de alfa de Cronbach

\begin{tabular}{|l|c|c|c|c|c|}
\hline \multicolumn{1}{|c|}{ Ítems de la escala GLIM } & $\mathbf{n}$ & $\begin{array}{c}\text { Correlación } \\
\text { elemento-total }\end{array}$ & $\begin{array}{c}\text { Correlación si se } \\
\text { elimina el elemento }\end{array}$ & $\begin{array}{c}\text { Covarianza media } \\
\text { entre elementos }\end{array}$ & $\begin{array}{c}\text { Alfa de Cronbach si se } \\
\text { elimina el elemento }\end{array}$ \\
\hline Pérdida de peso voluntaria & 123 & 0,7718 & 0,4960 & 0,1017 & 0,5662 \\
\hline Bajo IMC & 123 & 0,7037 & 0,4401 & 0,1275 & 0,5246 \\
\hline Masa muscular reducida & 123 & 0,8326 & 0,6151 & 0,0770 & 0,4147 \\
\hline $\begin{array}{l}\text { Ingesta alimentaria o } \\
\text { absorción reducida }\end{array}$ & 123 & 0,4416 & 0,2314 & 0,2028 & 0,6961 \\
\hline
\end{tabular}

Tabla 5. Resultados de validez, exactitud diagnóstica, seguridad y razón de probabilidad de los criterios GLIM usando VGS

\begin{tabular}{|c|c|c|c|}
\hline & Resultado & IC $95 \%$ & $\begin{array}{c}\text { Valor de referencia } \\
\text { aceptable }\end{array}$ \\
\hline Prueba kappa (sin DN, DN moderada, DN grave) & $0,5946(p<0,00001)$ & $0,46-0,52$ & $0,61-0,80$ \\
\hline Prueba kappa (sin DN, con DN) & $0,7777(p<0,00001)$ & $0,68-0,86$ & $0,61-0,80$ \\
\hline Sensibilidad & $99,1 \%$ & $95 \%-100 \%$ & $>80 \%$ \\
\hline Especificidad & $71,4 \%$ & $41,9 \%-91,6 \%$ & $>80 \%$ \\
\hline $\mathrm{LR}+$ & 3,47 & $1,51-7,94$ & $5-10$ \\
\hline LR - & 0,0128 & $0,018-0,929$ & $0,1-0,2$ \\
\hline VPP & $96,4 \%$ & $91,1 \%-99 \%$ & - \\
\hline VPN & $90,9 \%$ & $58,7 \%-99,8 \%$ & - \\
\hline
\end{tabular}

DN: desnutrición; IC: intervalo de confianza; LR: razón de verosimilitud; VPN: valor predictivo negativo; VPP: valor predictivo positivo.

La prevalencia de DN, a pesar de ser elevada por lo mencionado previamente, fue levemente superior utilizando el GLIM frente a VGS (91\% frente a $88 \%$ ). Brito y colaboradores obtuvieron resultados con igual tendencia $(41,6 \% \text { frente a } 33,9 \%)^{(10)}$. En discordancia, Allard y colaboradores hallaron un 33,29\% frente a $45,15 \%$, considerando como limitante que dicho estudio fue retrospectivo y utilizó únicamente los datos del GLIM que tuvieran disponibles ${ }^{(8)}$. El porcentaje de $\mathrm{DN}$ grave en el presente estudio resultó ampliamente mayor utilizando el GLIM (52,03 \% frente a $32,52 \%$ ) al igual que Allard y colaboradores $(19,77 \%$ frente a $11,73 \%)^{(8)}$.

Por una parte, al realizar el análisis factorial de cada criterio que compone el GLIM se concluye que, en caso de eliminar el ítem "ingesta reducida", la consistencia interna llegaría a ser considerada "buena" (cercana a 0,7 ). Por otra parte, se evidencia la importancia de la variable "masa muscular reducida", ya que sin la misma la consistencia interna resultaría aún menor (alfa:
0,4147). Por tal motivo, se requiere establecer puntos de corte y criterios operativos para la evaluación de la masa muscular. A pesar de que la antropometría presenta como limitante una menor confiabilidad con respecto a la bioimpedancia y absorciometría de energía dual con rayos $\mathrm{X}$, además del posible sesgo interobservador ${ }^{(34)}$, en la presente investigación no se contó con tal equipamiento. Es de importancia protocolizar un método de medición de la masa muscular accesible en la práctica clínica y estandarizar su uso a nivel global ${ }^{(7)}$.

En referencia a la fiabilidad del GLIM, las herramientas concuerdan significativamente $(p<0,00001)$ de forma moderada (0,5946, IC $95 \%$ : 0,46-0,72) al categorizar a los individuos en "sin DN", "DN moderada" y "DN grave". Si se considera el IC, se obtendría una buena fiabilidad ${ }^{(12)}$. La concordancia entre las herramientas es buena (0,7777, IC $95 \%: 0,68-0,86)$ al considerar individuos "con DN" y "sin DN".

La sensibilidad del GLIM en pacientes con riesgo nutricional $(99,1 \%)$ fue acorde a lo recomendado ( $\geq$ 
$80 \%)$ y se especula que el GLIM detecta fácilmente al individuo desnutrido ${ }^{(34)}$. Lo mismo fue demostrado por Brito y colaboradores $(86,6 \%$ ) y Burgel y colaboradores $(86,8 \%)^{(9,10)}$. En cambio, Allard y colaboradores encontraron una sensibilidad menor $(61,30 \%)^{(8)}$. Identificar a los pacientes con DN es sumamente importante. De lo contrario, no se realizaría el tratamiento nutricional adecuado, lo que supondría mayores complicaciones, peores resultados y un incremento de los costos sanitarios ${ }^{(37)}$. La especificidad del GLIM no alcanzó valores aceptables $(71,4 \%)$. Se considera que, al ser una herramienta de diagnóstico de DN, debería contar con alta especificidad para reducir el riesgo de catalogar como desnutrido a un individuo que no lo está. Los resultados del GLIM pueden diferir utilizando distintas herramientas de medición de masa muscular ${ }^{(20)}$. Por lo cual, como estrategias para lograr especificidad cercana a $80 \%$, se propone investigar cuál de las herramientas es la más específica y estandarizar su uso.

Las diferencias de sensibilidad y especificidad encontradas con otros estudios podrían deberse tanto a la disparidad en la metodología de los mismos como a la muestra de pacientes incluidos. A su vez, se debe considerar el desempeño de cada herramienta en las distintas poblaciones y las discrepancias en las técnicas de medición ${ }^{(8-10)}$.

La alta prevalencia de DN pudo haber influenciado el elevado VPP (96,4\%), dado que el mismo aumenta en condiciones de mayor prevalencia. El VPN también fue elevado, por lo que se puede concluir que de los pacientes sin DN según el GLIM solo el 9,1% sí presentaba DN según VGS. Ambos valores fueron más elevados en comparación con Allard y colaboradores. Las discrepancias podrían deberse a la diferencia en la prevalencia de DN de los estudios ${ }^{(8,38)}$.

La totalidad de la muestra en estudio presentó algún grado de inflamación o estrés, lo cual puede asociarse con la elevada sensibilidad y menor especificidad de la herramienta. Este hallazgo se relaciona con que no se utilizaron parámetros objetivos para su evaluación como las medidas de apoyo recomendadas (ejemplo: proteína C-reactiva $[\mathrm{PCR}])^{(8,20)}$.

Dentro de las limitaciones del presente estudio, además lo mencionado previamente sobre el uso de población tamizada con el SNAQ, se puede mencionar que la ingesta de alimentos fue obtenida mediante la percepción del paciente o su acompañante. En cuanto a la medición de la masa muscular, no se contó con la posibilidad de utilizar métodos de primera línea recomendados por GLIM, como son la tomografía axial computarizada (TAC) y la bioimpedancia, entre otros. No obstante, sí se utilizaron otras alternativas sencillas y de bajo costo también propuestas por el GLIM. Sumado a esto, hubiese sido de gran interés analizar cada una de las herramientas de medición de masa muscular utilizadas por separado observando el comportamiento del GLIM con cada una de ellas ${ }^{(7,34)}$.

Durante la realización de la investigación se hallaron algunas ventajas en el uso del GLIM en comparación con la VGS. Esta última incluye un examen físico exhaustivo y una evaluación de la capacidad funcional, los cuales requieren mayor tiempo y entrenamiento previo para realizarse adecuadamente.

Se considera de importancia resaltar que la redacción del protocolo de investigación y recolección de datos fue preliminar a la guía de validación de los criterios GLIM publicada por De van der Scuheren y el Grupo de Trabajo GLIM ${ }^{(34)}$. No obstante, se considera valioso su uso para el desarrollo de futuras investigaciones. Es importante destacar que, según los autores del GLIM, para estudios de validación de concordancia como este se debe considerar como método estándar de oro la valoración objetiva completa del estado nutricional.

Por último, mencionar que al haber incluido únicamente a aquellos pacientes que se encontraban en riesgo de $\mathrm{DN}$ y no a la totalidad de la población, los datos de este estudio podrían ser extrapolados únicamente a poblaciones con las mismas características y no a los pacientes hospitalizados en general.

Ante la falta de una herramienta estándar de oro y considerando al GLIM como una herramienta diagnóstica sencilla, rápida, objetiva y económica para evaluar el estado nutricional en la práctica clínica, se considera necesario realizar estudios adicionales de tipo multicéntricos y de seguimiento de cohorte para determinar la capacidad de los mismos en la predicción de resultados clínicos adversos (estancia hospitalaria, complicaciones clínicas y mortalidad), y de este modo determinar su validez predictiva. Para futuras investigaciones se plantea incluir a todos los pacientes hospitalizados sin excluir a aquellos sin riesgo de $\mathrm{DN}$, además de comparar los resultados obtenidos haciendo uso de los distintos métodos de medición de masa muscular, para establecer el más conveniente a utilizar en el ámbito clínico.

\section{CONCLUSIONES}

La herramienta GLIM, aplicada en pacientes con riesgo nutricional, posee una consistencia interna aceptable y una concordancia moderada o buena en comparación 
con la VGS, según la categorización utilizada. A su vez, posee una validez justa, con una sensibilidad mayor, pero una especificidad menor con respecto a la VGS. El GLIM logró una exactitud diagnóstica moderada en comparación con la VGS. Se requieren estudios de validez de la herramienta para su utilización en el ámbito hospitalario y, de este modo, lograr su aplicación a nivel mundial.

\section{PUNTOS CLAVE}

- Los criterios GLIM fueron propuestos con el fin de alcanzar un consenso mundial en la definición de los criterios diagnósticos de $\mathrm{DN}$ asociada con la enfermedad.

- El objetivo de este estudio es comparar la herramienta diagnóstica GLIM con la VGS en la práctica clínica con el fin de contribuir a su validación en Latinoamérica.

- El GLIM mostró una exactitud diagnóstica y una concordancia moderada o buena con respecto a la VGS al ser utilizada en pacientes con riesgo nutricional.

- La consistencia interna del GLIM mostró ser aceptable. Se vio que el ítem "masa muscular reducida" fue relevante, de modo que se sugieren futuras investigaciones que evalúen la aplicación de los distintos métodos de medición de masa muscular en los criterios GLIM.

- Como conclusión, la herramienta GLIM, aplicada en pacientes con riesgo nutricional, posee una validez justa con una mayor sensibilidad y una menor especificidad con respecto a la VGS.

\section{Agradecimientos}

Nuestros más sinceros agradecimientos a:

- La Lic. María Angélica Nadal, jefe del servicio de alimentación y dietoterapia del Hospital Universitario CEMIC.

- Las nutricionistas de planta del servicio de alimentación y dietoterapia del Hospital Universitario CEMIC.

- Las residentes en Nutrición del Instituto Universitario CEMIC.

- El Dr. Juan Gili y el Dr. Hugo Krupitzki, por el asesoramiento metodológico.

\section{Declaración de autoría}

P. Navarro, O. Capelli, J. Adaglio, R. Barritta contribuyeron igualmente a la concepción y diseño de investigación, adquisición, análisis e interpretación de los datos. P. Navarro y O. Capelli redactaron el manuscrito. Todos los autores revisaron el manuscrito, acuerdan ser plenamente responsables de garantizar la integridad y precisión del trabajo, y leyeron y aprobaron el manuscrito final.

\section{Conflicto de intereses}

Los autores declaran no tener ningún conflicto de intereses.

\section{Fuente de financiación}

El presente estudio no tuvo financiación.

\section{Referencias bibliográficas}

1. Correia MITD, Perman MI, Waitzberg DL. Hospital malnutrition in Latin America: A systematic review. Clin Nutr. 2017;36(4):958-967. doi: 10.1016/j.clnu.2016.06.025

2. Crivelli A, Perman M, Wyszynski D, Alomar F, Bellone M, De Loredo D, et al. Estudio AANEP 99: Prevalencia de desnutrición en hospitales de la Argentina [Internet]. RNC 2001;10:121-34 [consultado el 13 de mayo de 2021]. Disponible en: https://www.researchgate.net/ publication/261287437_99_PREVALENCIA_DE_ DESNUTRICION_EN_HOSPITALES_DE_LA_ARGENTINA_METODOLOGIA_DEL_ESTUDIO_Y_ ANALISIS_PRELIMINAR_DE_LOS_RESULTADOS

3. Canicoba M, Baptista G, Visconti G. Funciones y competencias del nutricionista clínico. Documento de consenso. Rev Cubana Aliment Nutr. 2013;23(1):146-72.

4. Epp LM, Salonen BR, Hurt RT, Mundi MS. Cross-sectional Evaluation of Home Enteral Nutrition Practice in the United States in the Context of the New Enteral Connectors. JPEN J Parenter Enteral Nutr. 2019;43(8):1020-1027. doi: 10.1002/ jpen. 1510

5. van Bokhorst-de van der Schueren MA, Guaitoli PR, Jansma EP, de Vet HC. Nutrition screening tools: does one size fit all? A systematic review of screening tools for the hospital setting. Clin Nutr. 2014;33(1):39-58. doi: 10.1016/j. clnu.2013.04.008

6. Barbosa-Silva MC, Barros AJ. Indications and limitations of the use of subjective global assessment in clinical practice: an update. Curr Opin Clin Nutr Metab Care. 2006;9(3):263-9. doi: 10.1097/01.mco.0000222109.53665.ed

7. Cederholm T, Jensen GL, Correia MITD, Gonzalez MC, Fukushima R, Higashiguchi T, et al. GLIM criteria for the diagnosis of malnutrition - A consensus report from the global clinical nutrition community. J Cachexia Sarcopenia Muscle. 2019;10(1):207-217. doi: 10.1002/jcsm.12383

8. Allard JP, Keller H, Gramlich L, Jeejeebhoy KN, Laporte M, Duerksen DR. GLIM criteria has fair sensitivity and speci- 
ficity for diagnosing malnutrition when using SGA as comparator. Clin Nutr. 2020;39(9):2771-2777. doi: 10.1016/j. clnu.2019.12.004

9. Burgel CF, Eckert IDC, Brito JE, Rodrigues FW, Silva FM. Accuracy of three tools for malnutrition diagnosis in hospitalised patients: Comparison to subjective global assessment. J Hum Nutr Diet. 2021. doi: 10.1111/jhn.12907

10. Brito JE, Burgel CF, Lima J, Chites VS, Saragiotto CB, Rabito EI, et al. GLIM criteria for malnutrition diagnosis of hospitalized patients presents satisfactory criterion validity: A prospective cohort study. Clin Nutr. 2021;40(6):4366-4372. doi: 10.1016/j.clnu.2021.01.009

11. Galindo Martín CA, Aportela Vázquez VA, Becerril Hernández F, Aguilar Medina CR, Ayala Carrillo SL, Chávez Flores A, et al. The GLIM criteria for adult malnutrition and its relation with adverse outcomes, a prospective observational study. Clin Nutr ESPEN. 2020;38:67-73. doi: 10.1016/j. clnesp.2020.06.015

12. Jiménez Villa J, Argimon Pallás JM. Tamaño de la muestra. En: Métodos de investigación clínica y epidemiológica. 4. ${ }^{a}$ edición. Barcelona: Elsevier; 2013. p. 142-154.

13. Hernández R, Fernández C, Baptista P. Muestreo en la investigación cualitativa. En: Metodología de la investigación. 6. ${ }^{\mathrm{a}}$ edición. México D.F. :McGraw-Hill Interamericana; 2014. p. 390-2.

14. Detsky AS, McLaughlin JR, Baker JP, Johnston N, Whittaker $\mathrm{S}$, Mendelson RA, et al. What is subjective global assessment of nutritional status? JPEN J Parenter Enteral Nutr. 1987;11(1):8-13. doi: 10.1177/014860718701100108

15. da Silva Fink J, Daniel de Mello P, Daniel de Mello E. Subjective global assessment of nutritional status - A systematic review of the literature. Clin Nutr. 2015;34(5):785-92. doi: 10.1016/j.clnu.2014.12.014

16. Normative grip strength data [internet]. Patterson Medical Jamar Smart User Manual-Hand dynamometer [consultado el 28 de julio de 2021]. Disponible en: https://www.manualslib.com/manual/1272692/Patterson-Medical-Jamar-Smart. html?page $=10 \#$ manual

17. Frisancho AR. New norms of upper limb fat and muscle areas for assessment of nutritional status. Am J Clin Nutr. 1981;34(11):2540-5. doi: 10.1093/ajcn/34.11.2540

18. Guastavino P, Llames L. Principios para la valoración del estado nutricional. En: Canicoba, M. Mauricio S (editores). Valoración del estado nutricional en diversas situaciones clínicas. 1. ${ }^{\text {a }}$ edición. Lima, Perú: Universidad Privada del Norte; 2017. p. 98-110.

19. Lera L, Sánchez H, Ángel B, Albala C. Mini Nutritional Assessment Short-Form: Validation in Five Latin American Cities. SABE Study. J Nutr Health Aging. 2016;20(8):797805. doi: 10.1007/s12603-016-0696-z

20. Contreras-Bolívar V, Sánchez-Torralvo FJ, Ruiz-Vico M, González-Almendros I, Barrios M, Padín S, Alba E, et al. GLIM Criteria Using Hand Grip Strength Adequately
Predict Six-Month Mortality in Cancer Inpatients. Nutrients. 2019;11(9):2043. doi: 10.3390/nu11092043

21. Sousa-Santos AR, Amaral TF. Differences in handgrip strength protocols to identify sarcopenia and frailty - a systematic review. BMC Geriatr. 2017;17(1):238. doi: 10.1186/s12877017-0625-y

22. Hogrel JY. Grip strength measured by high precision dynamometry in healthy subjects from 5 to 80 years. BMC Musculoskelet Disord. 2015;16:139. doi: 10.1186/s12891015-0612-4

23. Belcher HJCR, Smith H. Extended dynamometry: reference values. J Hand Surg Eur Vol. 2019;44(2):196-202. doi: $10.1177 / 1753193418805959$

24. Donis JH. Evaluación de la validez y confiabilidad de una prueba diagnóstica. Avances en Biomedicina. 2012;1(2):7381.

25. Goforth C. Using and Interpreting Cronbach's Alpha. Research Data Services and Sciences [Internet]. University of Virginia Library; 2015 [consultado el 13 de mayo de 2021]. Disponible en: https://data.library.virginia.edu/using-andinterpreting-cronbachs-alpha/

26. Carvajal A, Centeno C, Watson R, Martínez M, Sanz Rubiales Á. ¿Cómo validar un instrumento de medida de la salud?. Anales Sis San Navarra. 2011;34(1): 63-72.

27. Burgos ME, Manterola C. Cómo interpretar un artículo sobre pruebas diagnósticas. Revista Chilena de Cirugía. 2010;62(3):301-8. doi: 10.4067/S071840262010000300018

28. Jiménez Villa J, Argimon Pallás JM. Sensibilidad y especificidad. En: Métodos de investigación clínica y epidemiológica. 4. ${ }^{a}$ edición. Barcelona: Elsevier; 2013. p. 339-344.

29. Jiménez Villa J, Argimon Pallás JM. Análisis de concordancia. En: Métodos de investigación clínica y epidemiológica. $4 .{ }^{\mathrm{a}}$ edición. Barcelona: Elsevier; 2013. p. 345-349.

30. Power L, Mullally D, Gibney ER, Clarke M, Visser M, Volkert $\mathrm{D}$, et al. A review of the validity of malnutrition screening tools used in older adults in community and healthcare settings A MaNuEL study. Clin Nutr ESPEN. 2018;24:1-13. doi: 10.1016/j.clnesp.2018.02.005

31. Fuente-Alba CS, Molina Villagra M. Likelihood ratio (razón de verosimilitud): definición y aplicación en Radiología. Rev Argent Radiol. 2017;81(3):204-8. doi: 10.1016/j. rard.2016.11.002

32. Otero W, Pineda LF, Beltran LH. Utilidad de la razón de verosimilitud (likelihood ratio) en la práctica clínica. Rev Colomb Gastroenterol. 2001;16(1):33-36.

33. García GJJ. Significado y empleo de la razón de probabilidades en la práctica clínica. Rev Mex Pediatr. 2000;67(4);188-191.

34. de van der Schueren MAE, Keller H; GLIM Consortium, Cederholm T, Barazzoni R, Compher C, et al. Global Leadership Initiative on Malnutrition (GLIM): Guidance on validation of the operational criteria for the diagno- 
sis of protein-energy malnutrition in adults. Clin Nutr. 2020;39(9):2872-2880. doi: 10.1016/j.clnu.2019.12.022

35. Neelemaat F, Meijers J, Kruizenga $H$, van Ballegooijen $H$, van Bokhorst-de van der Schueren M. Comparison of five malnutrition screening tools in one hospital inpatient sample. J Clin Nurs. 2011;20(15-16):2144-52. doi: 10.1111/j.13652702.2010.03667.x

36. Kruizenga HM, Seidell JC, de Vet HC, Wierdsma NJ, van Bokhorst-de van der Schueren MA. Development and validation of a hospital screening tool for malnutrition: the short nutritional assessment questionnaire (SNAQ). Clin Nutr. 2005;24(1):75-82. doi: 10.1016/j.clnu.2004.07.015
37. Palma Milla S, Meneses D, Valero M, Calso M, García Vázquez N, Ruiz Garrido M, et al. Costes asociados a la desnutrición relacionada con la enfermedad y su tratamiento: revisión de la literatura. Nutr Hosp. 2018;35(2):442-460. doi: 10.20960/ nh.1204

38. Moriana M, Civera M, Artero A, Real JT, Caro J, Ascaso JF, et al. Validity of subjective global assessment as a screening method for hospital malnutrition. Prevalence of malnutrition in a tertiary hospital. Endocrinol Nutr. 2014;61(4):184-9. doi: 10.1016/j.endonu.2013.10.006 